CUBO A Mathematical Journal

Vol.20, $N^{\underline{O}} 01$, (65-78). March 2018

\title{
On rigid Hermitean lattices, II
}

\author{
Ana Cecilia de la MAZA \\ Departamento de Matemática y Estadística, \\ Universidad de la Frontera, \\ Temuco, Chile. \\ REMo MoRESI \\ Cerfim, \\ cp 1132, 6601 Locarno, Switzerland, \\ anace.delamaza@ufrontera.cl, romicatj@yahoo.it
}

\begin{abstract}
We study the indexed Hermitean lattice of type 0 generated by a single element a subjected to the relation $a \leq b^{\perp} \wedge b^{\perp}=0$. We prove that it is finite, provided that two crucial indices are finite. We show that index relations imply algebraic relations and describe the lattice by means of its subdirectly irreducible factors. We finally use the results to confirm a conjecture appeared in 2000.
\end{abstract}

\section{RESUMEN}

Estudiamos el reticulado Hermitiano finito indexado de tipo 0 generado por un solo elemento a sujeto a la relación $\mathrm{a} \leq \mathrm{b}^{\perp} \wedge \mathrm{bb}^{\perp}=0$. Probamos que es finito, suponiendo que dos índices cruciales son finitos. Mostramos que las relaciones de índices implican relaciones algebraicas y describimos el reticulado a travs de sus factores subdirectamente irreductibles. Finalmente, usamos nuestros resultados para confirmar una conjetura aparecida el ao 2000.

Keywords and Phrases: Lattices, semilattices, modular lattices, Hermitean lattices, orthogonal geometry.

2010 AMS Mathematics Subject Classification: 03G10,06A12, 06C05, 06B25. 


\section{Introduction}

The importance of lattices in infinite-dimensional orthogonal geometry was brought to attention by the pioneering work of Herbert Gross (1936-1989): see in particular G1 and G2. All examples treated in origin are sublattices of some $\mathcal{L}(\mathrm{E})$, the subspace-lattice of an $\boldsymbol{\aleph}_{0}$-dimensional vector space $E$ over an appropriate division ring $k$, together with the orthogonal operation induced by a Hermitean form $\phi$ (i.e. $X \mapsto X^{\perp}:=\{y \in E \mid \phi(y, x)=0 \forall x \in X\}$ ) and were used to study geometric invariants, for instance dimension of quotient spaces or intersections with the subspace $E^{*}$ of trace-valued vectors in $E$. The fact that $E^{*} \neq E$ only if $\operatorname{char}(k)=2$ was also playing some role. After some time of concrete investigations with subspace lattices (see e.g. [M1]), the natural idea to insert all considerations into an abstract setting gave rise to the following definitions (cf. [KKW], Ch. IV):

A Hermitean lattice (HL for short) is an algebra ( $\left.\mathrm{L}, 0,1, \cdot,+,{ }^{\perp}, \mathrm{b}\right)$ of type $\langle 0,0,2,2,1,0\rangle$ such that

i) $(\mathrm{L}, 0,1, \cdot,+)$ is a modular lattice with universal bounds 0,1 ;

ii) ${ }^{\perp}: \mathrm{L} \rightarrow \mathrm{L}$ is a unary operation with $1^{\perp}=0$ and

$$
x \leq\left(x^{\perp} y\right)^{\perp} \quad \forall x, y \in \mathrm{L}
$$

iii) $b \in L$ is a nullary operation with

$$
x x^{\perp} \leq \mathrm{b} \quad \forall x \in \mathrm{L} .
$$

In case $b$ is explicitly not trivial (i.e. $b \neq 1$ ), the modular law in $i$ ) is sometimes replaced by the stronger Fano identity

$$
(w+v)(y+z) \leq(w+y)(v+z)+(w+z)(v+y) .
$$

If we drop the operation "+", then we obtain the notion of Hermitean semilattice (HSL for short). In the present paper we will endow HL $\mathrm{L}$ with a so-called index function of type 0 (IF for short), i.e a function $\delta$ from the set of quotients of $L$ into the set of cardinals $\leq \boldsymbol{\aleph}_{\mathrm{o}}$, with the following properties:

$$
\begin{array}{r}
\delta(x / y) \geq \delta(x z / y z) \\
\delta(x / y) \geq \delta(x+z / y+z), \\
\delta(x / y) \geq \delta\left(y^{\perp} / x^{\perp}\right), \\
\delta(x / y)+\delta(y / z)=\delta(x / z), \\
\delta(x / y)=0 \Longleftrightarrow x=y .
\end{array}
$$


We will speak about indexed Hermitean lattices (IHL). By dropping (1.3), we obtain the notion of indexed Hermitean semilattices (IHSL).

A major task of the theory of $\mathrm{H}(\mathrm{S}) \mathrm{L}$ consists in describing the free objects $\mathrm{S}[\mathrm{a}]$ and $\mathrm{F}[\mathrm{a}]$, generated by a single element $a$ in the varieties of HSL and HL, respectively. Since such objects are infinite, a more realistic project consists in studying appropriate presentations under (index) relations suggested by geometrical choice (see [G1], [M1], [M2] and also the bibliography in [KKW] for many known examples). One of these options is given by the relation $a \leq b^{\perp}$, which was introduced in DM3 and gave rise to the concept of rigid $H(S) L$. Here we continue such investigation and consider rigid HL with the (somewhat complementary) property $b b^{\perp}=0$. In the above work the HSL $S:=S\left[a ; a \leq b^{\perp} \wedge b b^{\perp}=0\right]$ was already computed, but here we briefly reproduce its description, without proofs, to make this paper more self-contained. Since the corresponding HL is most probably infinite, we work with an IF $\delta$ and start our research with the following hypothesis:

$$
\delta\left(\mathrm{a}^{\perp} / \mathrm{d}_{1}^{\perp}\right)<\mathrm{\aleph}_{0} \wedge \delta\left(\mathrm{b}^{\perp} / \mathrm{c}_{1}^{\perp}\right)<\mathrm{\aleph}_{0}
$$

where

$$
c_{1}:=d^{\perp} e^{\perp}, d_{1}:=c^{\perp} e^{\perp} \text {, and } c:=a^{\perp} e^{\perp}, d:=b^{\perp} e^{\perp}, e:=a^{\perp} b^{\perp} .
$$

The algebraic relations forced by the index condition (1.7) are given below in (4.4), Theorem 4.1 . and have the following important consequence:

$F:=F[a ;(10 \wedge(4.4)]$ is finite and has 23 subdirectly irreducible factors.

The factors are listed in Tables II, III and IV, Section 7, together with the associated critical quotients.

We will finally use these results to confirm conjecture 2 in $\underline{\mathrm{M} 2}$ and to suggest an application in orthogonal geometry.

We conclude this introduction with two more remarks:

- Without (1.7), F would be most probably infinite (cf. also the arguments given in [M2]). Thus we can recognize the importance of the intervals $\left[d_{1}^{\perp}, a^{\perp}\right]$ and $\left[c_{1}^{\perp}, b^{\perp}\right]$ in the above HL. Moreover, it is easy to prove that (1.7) is a weakening of the condition $\delta(1 / \mathrm{b})<\boldsymbol{\aleph}_{0}$, which has a natural interpretation in orthogonal geometry (cf. Section 6 ) and was used as hypothesis in many precedent investigations.

- S appeared naturally as substructure in other works (see [M2] and DM2]). This important fact was an additional motivation for the present study.

\section{$2 \quad$ Preliminaries}

Lemma 2.1. Any countable $H L$ is indexable. 
Proof. Each HL admits the trivial IF, defined to have value $\boldsymbol{\aleph}_{0}$ on each nontrivial quotient.

Lemma 2.2. The class of IHL is closed with respect to subalgebras, homomorphic images and countable products.

Proof. This is just a slight generalization of Proposition 21 in [KKW], Ch. IV.

Clearly, the existence of a nontrivial IF on some HL is controlled by prime quotients. Our lattices do not present difficulties such as described in $[\mathrm{S}$ because the subdirectly irreducible factors are finite and known.

The next result represents the key to obtain algebraic relations from index relations (cf. proof of Theorem 4.1):

Lemma 2.3. Let $\mathfrak{u} / v$ be any finite quotient of an IHL. If $v=v^{\Perp}$ then $\mathfrak{u}=\mathfrak{u}^{\Perp}$.

Proof. $\delta(u / v) \geq \delta\left(v^{\perp} / u^{\perp}\right) \geq \delta\left(u^{\Perp} / v^{\Perp}\right)=\delta\left(u^{\Perp} / v\right) \geq \delta(u / v)$.

For the sake of precision we give also the following

Definition 2.4. $S\left[a: a \leq b^{\perp}\right]$ is the initial object of the class of rigid HSL. Similarly, $F\left[a: a \leq b^{\perp}\right]$ is the initial object of the class of rigid $H L$.

Thus any rigid $H(S) L$ is a homomorphic image of the initial object.

We could have been even more precise by saying that this is in fact the concept of a 1-generated rigid $H(S) L$, a special case of $n$-generated rigid $H(S) L$, but of course, for the moment, all this is not necessary.

We conclude this section by remarking that the axiom (1.1) is equivalent with the following conditions:

(i) $x \leq x^{\Perp} ; \quad$ (ii) $x \leq y \Rightarrow y^{\perp} \leq x^{\perp}$.

This may facilitate some computations.

\section{Description of $S$}

Theorem 3.1. The HSL S has 18 elements and its structure is given by the diagram depicted in Figure 1 (see Section 7).

Proof. See DM3. 
Since we are interested in indices, we consider an IF $\delta$ on $S$ and put

$$
\begin{array}{llll}
\beta_{1}:=\delta(a / 0), & \beta_{2}:=\delta(b / 0), & \beta_{3}:=\delta(e / 0), & \beta_{4}:=\delta\left(c / c_{1}\right), \\
\beta_{5}:=\delta\left(a^{\Perp} / a\right), & \beta_{6}:=\delta\left(b^{\Perp} / b\right), & \beta_{7}:=\delta\left(c_{1} / b^{\Perp}\right), & \beta_{8}:=\delta\left(d_{1} / a^{\Perp}\right) .
\end{array}
$$

Theorem 3.2. (Relations among indices in $\mathrm{S}$ )

(i) All other indices of $\mathrm{S}$ are determined by $\beta_{1}, \cdots, \beta_{8}$ as is shown in

Figure 2, Section \%.

(ii) In particular, the following relations hold:

a) $\beta_{4} \neq 0$ implies $\beta_{1}=\beta_{2}=\aleph_{0}$;

b) $\beta_{5} \neq 0$ implies $\beta_{1}=\aleph_{0}$;

c) $\beta_{6} \neq 0$ implies $\beta_{2}=\aleph_{0}$;

d) $\beta_{7} \neq 0$ or $\beta_{8} \neq 0$ implies $\beta_{1}=\beta_{2}=\beta_{3}=\aleph_{0}$.

Proof. See [DM3].

Remark 3.3. Using the above Theorem, we find 8 subdirectly irreducible factors of S. They are reproduced in Tables I and II, Section $\%$.

\section{Description of $F$}

Remembering (1.8), let us consider the two descending chains $\left\{a_{1}, a_{2}, a_{3}\right\}:=\left\{a^{\perp}, d_{1}^{\perp}, d^{\perp}\right\}$ and $\left\{b_{1}, b_{2}, b_{3}\right\}:=\left\{b^{\perp}, c_{1}^{\perp}, c^{\perp}\right\}$.

For $1 \leq i, j \leq 3$ we define

$$
a_{i j}:=a_{i}\left(b_{j}+e^{\perp}\right), \quad b_{i j}:=b_{j}\left(a_{i}+e^{\perp}\right), \quad e_{i j}:=e^{\perp}\left(a_{i}+b_{j}\right) .
$$

Let $I_{1}, I_{2}$ and $I_{3}$ be the modular sublattices of $F$ generated by $\left\{a^{\perp}, d_{1}^{\perp}, d^{\perp}, c, b_{31}^{\perp}, b_{21}^{\perp}, b_{11}^{\perp}, b, e\right\} \cup\left\{a_{i j}\right\},\left\{b^{\perp}, c_{1}^{\perp}, c^{\perp}, d, a_{13}^{\perp}, a_{12}^{\perp}, a_{11}^{\perp}, a, e\right\} \cup\left\{b_{i j}\right\}$ and $\left\{e^{\perp}, c, c_{1}, b, b_{31}^{\perp}, b_{21}^{\perp}, b_{11}^{\perp}, d, d_{1}, a_{13}^{\perp}\right.$, $\left\{e_{i j}\right\}$, respectively.

By the main result in DM1, $I_{1}, I_{2}$ and $I_{3}$ coincide with the principal ideals of $F_{0}:=<$ $I_{1} \cup I_{2} \cup I_{3}>$ generated by $a^{\perp}, b^{\perp}$ and $e^{\perp}$ respectively. Moreover, they are distributive and additively generate $F_{0}$. We want to show that $F_{0}=F$.

To this end it will be useful to define the following indices: 
$\alpha_{i}:=\beta_{i}$ for $i=1,2,3,4,5,6$ and further

$$
\begin{array}{lll}
\alpha_{7}:=\delta\left(e^{\perp} / e_{11}\right), & \alpha_{8}:=\delta\left(c^{\perp} / b_{13}\right), & \alpha_{9}:=\delta\left(d^{\perp} / a_{31}\right), \\
\alpha_{10}:=\delta\left(1 / a^{\perp}+b^{\perp}+e^{\perp}\right), & \alpha_{11}:=\delta\left(b_{11}^{\perp} / b^{\Perp}\right), & \alpha_{12}:=\delta\left(a_{11}^{\perp} / a^{\perp}\right), \\
\alpha_{13}:=\delta\left(d_{1}^{\perp} / a_{21}+d^{\perp}\right), & \alpha_{14}:=\delta\left(c_{1}^{\perp} / b_{12}+c^{\perp}\right), & \alpha_{15}:=\delta\left(b_{33} / d_{1}+e\right), \\
\alpha_{16}:=\delta\left(d_{1} / a_{13}^{\perp}\right), & \alpha_{17}:=\delta\left(c_{1} / b_{31}^{\perp}\right), & \alpha_{18}:=\delta\left(a_{22} / a_{23}+a_{32}\right), \\
\alpha_{19}:=\delta\left(a_{12}^{\perp} / a_{11}^{\perp}\right), & \alpha_{20}:=\delta\left(a_{13}^{\perp} / a_{12}^{\perp}\right), & \alpha_{21}:=\delta\left(b_{31}^{\perp} / b_{21}^{\perp}\right), \\
\alpha_{22}:=\delta\left(b_{23} / b_{33}\right), & \alpha_{23}:=\delta\left(a_{32} / a_{33}\right) . &
\end{array}
$$

Theorem 4.1. (Description of $\mathrm{I}_{1}, \mathrm{I}_{2}$ and $\mathrm{I}_{3}$ in $\left.\mathrm{F}\right)$ :

1) The plain structure of $\mathrm{I}_{1}, \mathrm{I}_{2}$ and $\mathrm{I}_{3}$ is represented by the diagrams depicted in Fig 3, Fig 4 and Fig 5 of Section 7.

2) The ideals are connected by the following relations between indices:

$$
\begin{aligned}
\alpha_{4} & =\delta\left(d / d_{1}\right)=\delta\left(c / c_{1}\right), \\
\alpha_{11} & =\delta\left(b_{11}^{\perp} / b^{\Perp}\right)=\delta\left(b^{\perp} / b_{11}^{\Perp}\right), \\
\alpha_{12} & =\delta\left(a_{11}^{\perp} / a^{\Perp}\right)=\delta\left(a^{\perp} / a_{11}^{\Perp}\right), \\
\alpha_{15} & =\delta\left(b_{33} / d_{1}+e\right)=\delta\left(a_{33} / c_{1}+e\right)=\delta\left(e_{33} / c_{1}+d_{1}\right), \\
\alpha_{16} & =\delta\left(d_{1} / a_{13}^{\perp}\right)=\delta\left(a_{13} / a_{23}\right)=\delta\left(b_{13} / b_{23}\right)=\delta\left(e_{13} / e_{23}\right), \\
\alpha_{17} & =\delta\left(c_{1} / b_{31}^{\perp}\right)=\delta\left(a_{31} / a_{32}\right)=\delta\left(b_{31} / b_{32}\right)=\delta\left(e_{31} / e_{32}\right), \\
\alpha_{18} & =\delta\left(a_{22} / a_{23}+a_{32}\right)=\delta\left(e_{22} / e_{23}+e_{32}\right), \\
\alpha_{19} & =\delta\left(a_{12}^{\perp} / a_{11}^{\perp}\right)=\delta\left(b_{21}^{\perp} / b_{11}^{\perp}\right)=\delta\left(e_{11} / e_{12}+e_{21}\right), \\
\alpha_{20} & =\delta\left(a_{13}^{\perp} / a_{12}^{\perp}\right)=\delta\left(a_{12} / a_{13}+a_{22}\right)=\delta\left(b_{12} / b_{13}+b_{22}\right)=\delta\left(e_{12} / e_{13}+e_{22}\right), \\
\alpha_{21} & =\delta\left(b_{31}^{\perp} / b_{21}^{\perp}\right)=\delta\left(a_{21} / a_{22}+a_{31}\right)=\delta\left(b_{21} / b_{22}+b_{31}\right)=\delta\left(e_{21} / e_{22}+e_{31}\right), \\
\alpha_{22} & =\delta\left(b_{23} / b_{33}\right)=\delta\left(a_{23} / c+a_{33}\right), \\
\alpha_{23} & =\delta\left(a_{32} / a_{33}\right)=\delta\left(b_{32} / b_{33}+d\right)=\delta\left(e_{32} / d+e_{33}\right) .
\end{aligned}
$$

3) $\mathrm{I}_{1} \cup \mathrm{I}_{2} \cup \mathrm{I}_{3}$ is orthogonally closed in force of the following relations:

$$
\begin{array}{lll}
a_{11}+d_{1}^{\perp}=a_{11}^{\Perp}, & a_{12}+d_{1}^{\perp}=a_{12}^{\Perp}, & a_{13}+d_{1}^{\perp}=a_{13}^{\Perp}, \\
b_{11}+c_{1}^{\perp}=b_{11}^{\Perp}, & b_{21}+c_{1}^{\perp}=b_{21}^{\Perp}, & b_{31}+c_{1}^{\perp}=b_{31}^{\Perp} .
\end{array}
$$

Proof. 1) This is routine verification.

2) $\delta\left(d / d_{1}\right) \geq \delta\left(d_{1}^{\perp} / d^{\perp}\right) \geq \delta\left(d_{1}^{\perp} e^{\perp} / d^{\perp} e^{\perp}\right) \geq \delta\left(c / c_{1}\right) \geq \delta\left(c_{1}^{\perp} / c^{\perp}\right) \geq$ $\delta\left(c_{1}^{\perp} e^{\perp} / c^{\perp} e^{\perp}\right) \geq \delta\left(d / d_{1}\right)$. This shows the first equality. The second and third ones are evident. As to the fourth, just consider the free modular lattice generated by the triple $\left(d^{\perp}, c^{\perp}, e^{\perp}\right)$. The other equalities are proved analogously. 
3) We just show the first equality (the others follow in the same manner):

$$
\left.\delta\left(a_{11}+d_{1}^{\perp} / d_{1}^{\perp}\right)=\delta\left(a_{11} / a_{11} d_{1}^{\perp}\right)=\delta\left(a_{11} / a_{21}\right) \leq \delta\left(a^{\perp} / d_{1}^{\perp}\right)<\aleph_{0} \text { (by (1.7) }\right) .
$$

Thus $a_{11}+d_{1}^{\perp}=\left(a_{11}+d_{1}^{\perp}\right)^{\Perp}$ by Lemma 2.3 , because $d_{1}^{\perp}=\left(d_{1}^{\perp}\right)^{\Perp}$.

Since $d_{1}^{\perp} \leq a_{11}^{\Perp}$ (because $c+e \leq a_{11}$ ), we obtain the desired equality.

The rest is easy and it follows $F=F_{0}$.

Theorem 4.2. (Forced relations among indices):

i) If $\alpha_{7} \neq 0$ then $\alpha_{1}=\alpha_{2}=\aleph_{0}$;

ii) If $\alpha_{8} \neq 0$ then $\alpha_{1}=\alpha_{3}=\aleph_{0}$;

iii) If $\alpha_{9} \neq 0$ then $\alpha_{2}=\alpha_{3}=\Sigma_{0}$;

iv) For $i \in\{10,11,12,15,16,17,19\}$, if $\alpha_{i} \neq 0$ then $\alpha_{1}=\alpha_{2}=\alpha_{3}=\aleph_{0}$;

v) For $i \in\{13,14,18,20,21,22,23\}$, if $\alpha_{i} \neq 0$ then $\alpha_{1}=\alpha_{2}=\alpha_{3}=\alpha_{4}=\aleph_{0}$;

vi) $\alpha_{11}+\alpha_{12}+\alpha_{16}+\alpha_{17}+\alpha_{19}+\alpha_{20}+\alpha_{21}<\aleph_{0}$

Proof. Each implication follows in a way as was shown in the proof of Theorem 3.2, possibly in conjunction with Lemma 2.3.

The well known rule $(x+y)^{\perp}=x^{\perp} y^{\perp}$ may also be useful for computations. The last assertion is just the translation of (1.7) in terms of the indices $\alpha_{i}$.

\section{The subdirectly irreducible factors of F.}

In order to discover the factors of $F$ it is sufficient to work out $I_{1}, I_{2}$ and $I_{3}$ at the same time, using the relations given in Theorem 4.1 and Theorem 4.2.

The essence of the procedure consists in collecting all prime quotients that are connected with a given one via the algebraic operations: this will produce automatically the corresponding subdirectly irreducible factor, together with the associated relation.

Observe how useful are indices in this procedure: on the one hand they are associated in natural way to congruences, on the other hand the forced relations among them give directly the non minimal congruences in the subdirectly irreducible factors.

A little final caution is needed: there is a quotient which does not appear in the ideals, namely $1 /\left(a^{\perp}+b^{\perp}+e^{\perp}\right)$ (see the factor corresponding to $\alpha_{9}$ in Table III). Since $\left(a^{\perp}+b^{\perp}\right)^{\Perp}=\left(a^{\perp}+e^{\perp}\right)^{\Perp}=$ $\left(e^{\perp}+b^{\perp}\right)^{\Perp}=1$ this is the only exception.

The factors are labelled from 1 to 23 in Tables II, III and IV. The last table contains all non distributive members. 
Remark 5.1. From all the above results we deduce in particular that Conjecture 2 in [M2] is true: in fact, the finite codimensions indicated in the conjecture correspond to the ones given by (1.7).

Remark 5.2. There are plain lattice isomorphisms between different factors. Nevertheless we chose to give explicitly all diagrams, in order to facilitate visualization. It is worth noticing that the majority of this plain isomorphisms are induced by the map $\mathrm{a} \mapsto \mathrm{b}$ and $\mathrm{b} \mapsto \mathrm{a}$, which defines an involution of $\mathrm{S}$ that extends naturally to F. More precisely, there are eight pairs of symmetric factors, namely (1,2), (5,6), (8,9), (11,12), (13,14), (16,17), (20,21) and (2,23), all other factors being self symmetric.

\section{Remarks concerning applications to Hermitean spaces}

It is possible to prove that all factors of $F$ are implemented by Hermitean models. Hence they can be used to describe the congruence class of a subspace $A$ in a Hermitean space $(E, \phi)$ of denumerable dimension under the starting assumptions, where $A, E, E^{*}$ correspond to $a, 1, b$, respectively.

In general, these IHL will not suffice to build a complete set of geometric invariants, but they constitute a very important part. Details on these aspects cannot be discussed in the present work.

\section{Diagrams}

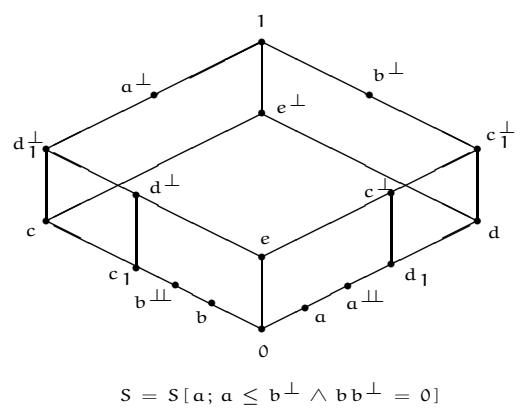

Figure 1

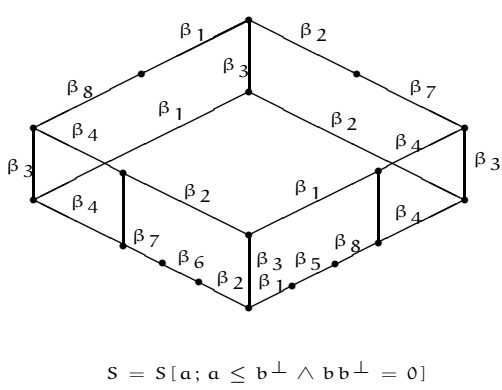

Figure 2 

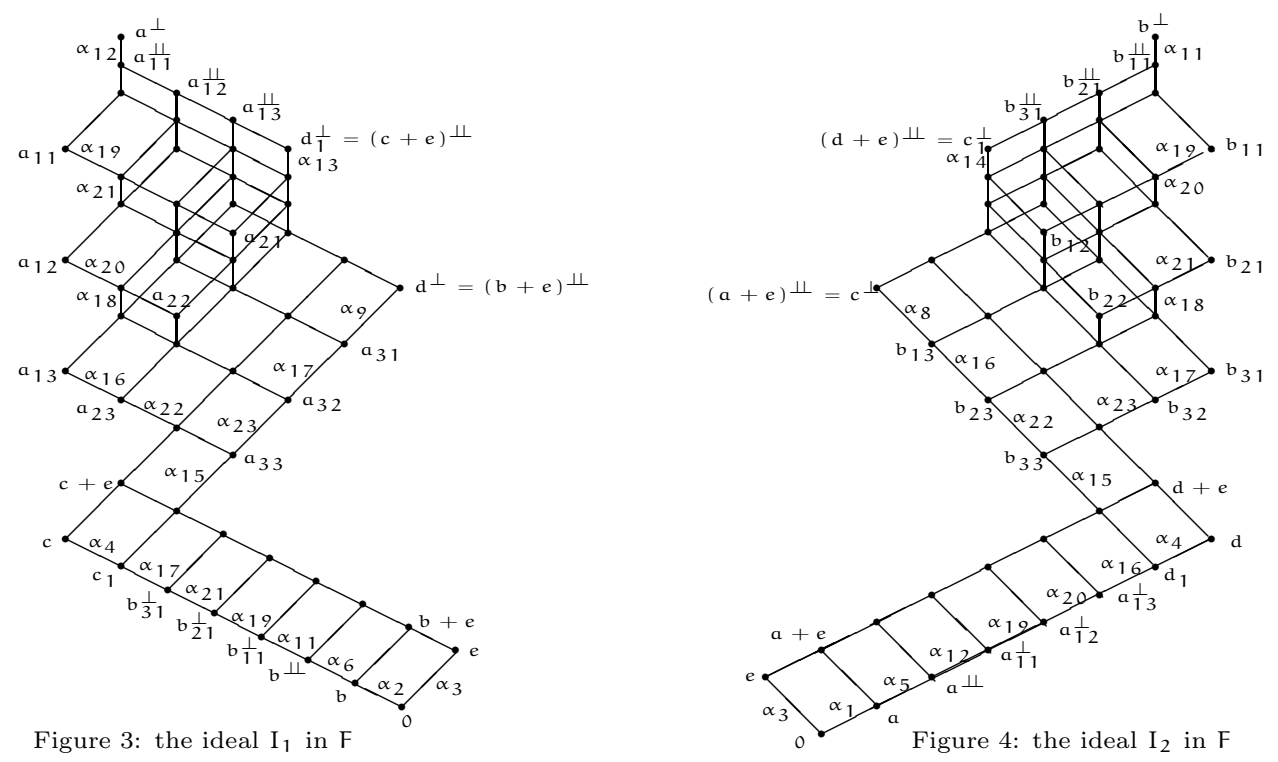

Figure 3: the ideal $\mathrm{I}_{1}$ in $\mathrm{F}$ 


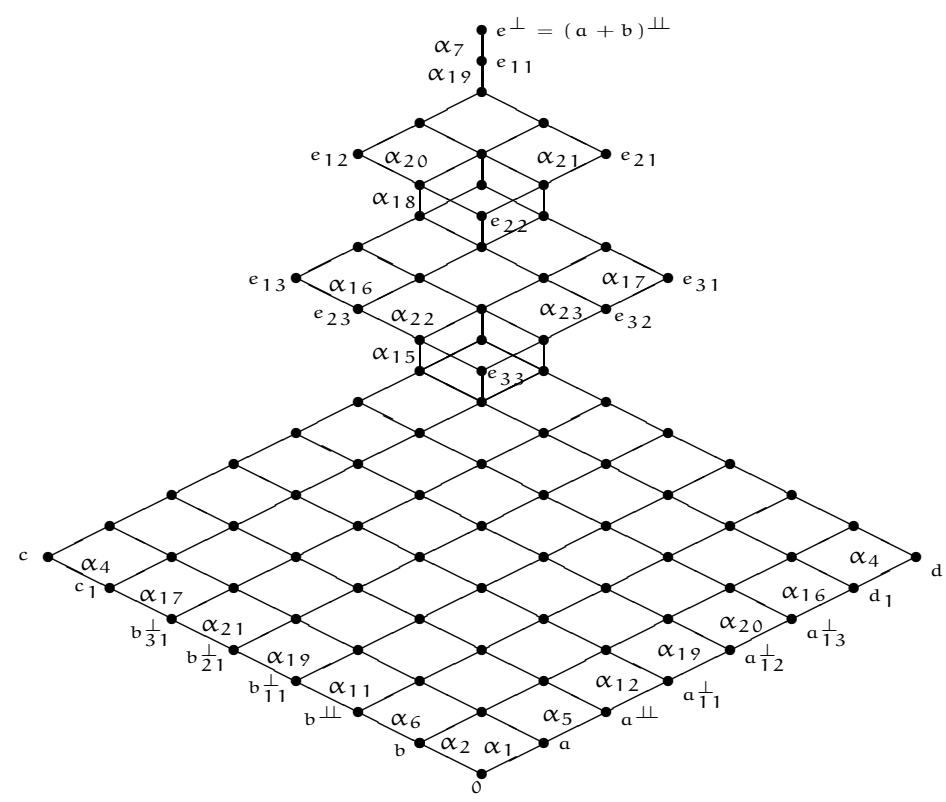

Figure 5: the ideal $\mathrm{I}_{3}$ in $\mathrm{F}$

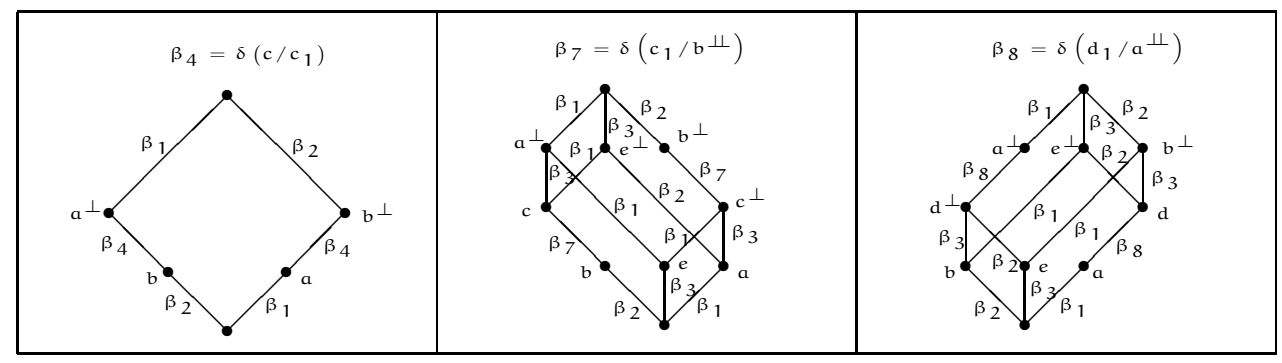




\begin{tabular}{|c|c|c|c|c|}
\hline $\begin{array}{c}\beta_{1}=\alpha_{1}=\delta(a / 0) \\
\alpha_{1} \varliminf_{b}^{a}\end{array}$ & $\begin{array}{c}\beta_{2}=\alpha_{2}=\delta(b / 0) \\
\alpha_{2} \prod_{a}^{b}\end{array}$ & $\begin{array}{c}\beta_{3}=\alpha_{3}=\delta(e / 0) \\
\alpha_{3} \prod_{b}^{e}\end{array}$ & $\begin{array}{rl}\beta_{5}= & \alpha_{5}=\delta(a \Perp / a) \\
& \alpha_{5} a^{a}{ }^{a} \\
\alpha_{1} & a \\
a^{\perp} & b \\
\alpha_{1}=x_{0} & \end{array}$ & 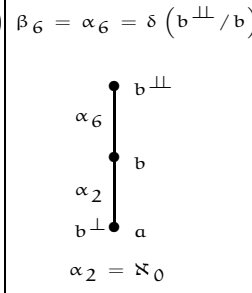 \\
\hline
\end{tabular}




\begin{tabular}{|c|c|c|}
\hline$\alpha_{1}=\alpha_{2}=\alpha_{0}$ & $\alpha_{7}=\delta\left(e^{\perp} / e_{11}\right)$ & $\alpha_{8}=\delta\left(c^{\perp} / b_{13}\right)$ \\
\hline $\begin{array}{c}\alpha_{9}=\delta\left(d^{\perp} / a_{31}\right) \\
\alpha_{2}=\alpha_{3}=x_{0}\end{array}$ & $\begin{array}{c}\alpha_{10}=\delta\left(\frac{1}{a^{\perp}+b^{\perp}+e^{\perp}}\right) \\
\alpha_{1}=\alpha_{2}=\alpha_{3}=\alpha_{0}\end{array}$ & $\begin{array}{l}\alpha_{11}=\delta\left(b \frac{1}{11} / b^{\Perp}\right) \\
\alpha_{1}=\alpha_{2}=\alpha_{3}=\alpha_{0}\end{array}$ \\
\hline$\alpha_{1}=\alpha_{2}=\alpha_{3}=\alpha_{0}$ & $\alpha_{13}=\delta\left(\frac{\mathrm{d}_{1}^{\perp}}{\mathrm{a}_{21}+\mathrm{d}^{\perp}}\right)$ & $\alpha_{1}=\alpha_{2}=\alpha_{3}=\alpha_{4}=\alpha_{0}$ \\
\hline
\end{tabular}


Table IV

$\left.\begin{array}{ll}\mathrm{b}_{33} \\ \mathrm{a}_{1}+\mathrm{e}\end{array}\right)$

\section{References}

[DM1] A.C. de la Maza, R.Moresi, On modular lattices generated by chains, Algebra Universalis 54 (2005), 475-488.

[DM2] A.C. de la Maza, R.Moresi, Hermitean (semi) lattices and Rolf's lattice, Algebra Universalis 66 (2011), 49-62.

[DM3] A.C. de la Maza, R.Moresi, On rigid Hermitean lattices, I, Preprint.

[G1] H. Gross, Quadratic forms in infinite dimensional vector spaces, Birkäuser, Boston, 1979.

[G2] H. Gross, Lattices and infinite-dimensional forms. "The lattice method", Order 4 (1987), 233-256. 
[KKW] H. A. Keller, U.-M. Künzi, M. Wild (eds), Orthogonal geometry in infinite dimensional vector spaces, Heft 53, Bayreuther Mathematische Schriften, Bayreuth, 1998.

[M1] R. Moresi, Modular lattices and Hermitean forms, Algebra Universalis 22 (1986), 279-297.

[M2] R. Moresi, A test-example of a quadratic lattice, Order 17 (2000), 215-226.

[R] H. L. Rolf, The free lattice generated by a set of chains, Pacific J. Math. 8 (1958), 585-595.

[S] E. T. Schmidt, On finitely generated simple modular lattice, Periodica Mathematica Hungarica 6(3) (1975), 213-216. 